\title{
Efficiency of Using Different Greater Taper Gutta-Percha Cones in Continuous Warm Vertical Condensation: An Ex Vivo Study
}

\author{
Reem Barakat ${ }^{1}$, Rahaf Almohareb ${ }^{2}$, Mamata Hebbal ${ }^{3}$, Ghada Alaskar ${ }^{4}$, Lama Alghufaily ${ }^{5}$, Nouf AlFarraj ${ }^{6}$, Alia Albaz $^{7}$
}

\begin{abstract}
Aim: The aim of this ex vivo randomized study is to evaluate the efficiency of gutta-percha cones that match a nickel-titanium instrumentation system and nonmatching greater taper cones, when used with continuous warm vertical condensation technique.

Material and methods: Thirty-six straight canals were prepared using ProTaper Next files, and the apical third was obturated using either ProTaperNext cones (group A), ISO uniform greater taper cones (group B), or nonstandardized cones (group C). Cone adaptation time was quantified by the number of required modifications. Micro-computed tomography was used to measure voids and sealer percentage.

Results: There was no significant difference between the groups regarding void volume $(p=0.666)$, percentage $(p=0.379)$, and the number of modifications $(p=0.757)$. Sealer percentage, however, was significantly lower in group B when compared to group A $(p=0.0194)$.

Conclusion: In straight canals, matching gutta-percha cones were not associated with significantly better obturation or saving time to fit the cone. Clinical significance: Using gutta-percha cones that do not match a nickel-titanium instrumentation system to obturate the straight canals with continuous warm vertical condensation technique is as efficient as using matching cones in terms of obturation quality and ease of cone fit. Keywords: Apical seal, Ex vivo, Gutta-percha cones, Obturation, ProTaper next, Sealer, Taper, Voids, Warm vertical condensation.

The Journal of Contemporary Dental Practice (2021): 10.5005/jp-journals-10024-3035
\end{abstract}

\section{INTRODUCTION}

A major factor that influences the success of endodontic therapy is achieving adequate apical seal, which depends on how well an obturation technique can adapt inert gutta-percha (GP) combined with a minimal amount of resorbable sealer, to the canal walls. ${ }^{1}$ Warm vertical condensation technique can compact heated GP into the apical third, adapting it to canal irregularities. ${ }^{2}$ A recent, simpler, less time-consuming, and periodontally safer version of warm vertical condensation, continuous warm vertical condensation (CWC), ${ }^{3}$ has shown superiority in terms of packing the canal with more $\mathrm{GPs}^{4}{ }^{4}$ and filling the canal-wall concavities ${ }^{5,6}$ and lateral canals. ${ }^{7}$

Before the introduction of nickel-titanium (NiTi) rotary instrumentation, nonstandardized GP cones (sizes fine-medium or medium) were the only GP cones available for use with warm vertical condensation. The NiTi alloy made it possible to fabricate either a uniform or variable greater taper root canal preparation instruments that can produce a more predictable apical third shape and taper. This has led to the evolution of greater taper GP cones that match the shape of these NiTi instruments. ${ }^{8}$ There are two categories of greater taper GP cones: uniform and nonuniform (variable) taper. The former is ISO standardized 0.04 to 0.06 tapered cones, while the latter is associated with a brand name specific to a NiTi instrumentation technique, as is the case for ProTaper Next. ${ }^{9}$

ProTaper Next are fifth-generation NiTi-shaping files made with M-wire technology and an offset design, which gives the system superior shaping capabilities due to its increased flexibility and resistance to fracture. ${ }^{10,11}$ The system has five shaping files $X 1$ to X5, corresponding to sizes $17 / 04,25 / 06,30 / 07,40 / 06$, and 50/06, respectively. ProTaper Next GP cones X1 to X5 that match the nonuniform taper of the ProTaper Next NiTi files are available for root canal obturation. \begin{tabular}{l}
\hline \hline${ }^{1,2}$ Clinical Dental Sciences Department, College of Dentistry, Princess \\
Nourah Bint Abdulrahman University, Riyadh, Kingdom of Saudi Arabia \\
${ }^{3}$ Department of Preventive Dental Science, College of Dentistry, \\
Princess Nourah Bint Abdulrahman University, Riyadh, Kingdom of \\
Saudi Arabia \\
${ }^{4-7}$ College of Dentistry, Princess Nourah Bint Abdulrahman University, \\
Riyadh, Kingdom of Saudi Arabia
\end{tabular}

Corresponding Author: Rahaf Almohareb, Clinical Dental Sciences Department, College of Dentistry, Princess Nourah Bint Abdulrahman University, Riyadh, Kingdom of Saudi Arabia, Phone: +966 559594130, e-mail: raalmohareb@pnu.edu.sa, almohareb.r@hotmail.com

How to cite this article: Barakat R, Almohareb R, Hebbal M, et al. Efficiency of Using Different Greater Taper Gutta-Percha Cones in Continuous Warm Vertical Condensation: An Ex Vivo Study. J Contemp Dent Pract 2021;22(1):56-61.

Source of support: This research was funded by the Deanship of Scientific Research at Princess Nourah Bint Abdulrahman University through the Fast-track Research Funding Program.

Conflict of interest: None

Manufacturers claim that these matching GP cones make root canal treatment less time-consuming, by allowing simple straightforward adaptation of the cones in the root canals and equally providing an effective seal. ${ }^{12}$ They market these matching GP cones at more expensive prices. For example, in Saudi Arabia, a single ProTaper Next GP cone costs 3 times the price of an ISO uniform greater taper GP cone and 15 times the price of a nonstandardized GP cone. ${ }^{13}$

While many studies compared the efficacy of using different types of GP cones with a single cone and lateral condensation 
techniques, ${ }^{12,14-16}$ the efficiency of their use with the CWC technique has not been investigated. Efficiency denotes achieving optimum results, e.g., superior canal obturation using the least amount of inputs, such as modifications, to adapt the cone into the canal. It signifies reducing the number of unnecessary resources (personal time and energy) used to produce the desired results. ${ }^{17}$

Therefore, the aim of this study is to evaluate the efficiency of using nonstandardized, ISO uniform greater taper and matching ProTaper Next GP cones with CWC technique in an ex vivo setting, which compares: (1) Quality of apical third obturation - defined by the volume of voids and percentage of sealer using the highresolution micro-computed tomography (micro-CT). (2) Time required to fit the cone in the canal. The null hypothesis was that there would be no difference between the three GP cone types.

\section{Materials and Methods}

\section{Sample Selection}

This study was conducted at Princess Nourah Bint Abdulrahman University (PNU), Dental College Simulation Lab, and King Saud University, Engineer Abdullah Bugshan Research Chair for Dental and Oral Rehabilitation Lab. Ethical approval for the study was obtained from the PNU Institutional Review Board.

Thirty-six extracted single-rooted teeth were collected from different private or governmental clinics in Riyadh, KSA. The teeth had been extracted for orthodontic or periodontal reasons. The sample size was based on previous studies, evaluating root canal quality using micro-CT. ${ }^{1,4,18-23}$ The teeth were stored for two days at room temperature in $3 \%$ sodium hypochlorite $(\mathrm{NaOCl})$ to remove organic debris. Subsequently, they were debrided with ultrasonic scalers, washed with distilled water, and then immersed in $10 \%$ formalin solution. The criteria for tooth selection included single straight root canal, no visible root caries or fractures/cracks on examination under the operating microscope ( $\mathrm{A} 3$ series; Global Surgical Corporation, USA), no signs of internal or external resorption or calcification, a completely formed apex. Teeth with excessively short roots were also excluded. Preoperative radiographs were taken to confirm canal anatomy. The buccolingual to mesiodistal diameter ratio was measured and selected to be $<2$ when examined at $5 \mathrm{~mm}$ from the apex.

\section{Root Canal Preparation}

Access cavities were prepared using endodontic access burs. A size 10 K-file (Medin, Czech Republic) was introduced into the canal until it was visible at the apical foramen. This was verified with the aid of dental loupes X3 (JTL Gobiz, Korea). Determination of working length was done by subtracting $0.5 \mathrm{~mm}$ from this measurement. This same file was used as a patency file during canal preparation. Only root canals, in which the first file fitted to the working length was a maximum size $20 \mathrm{~K}$-file, were included in this study.

Root canals were prepared using ProTaper Next files (Dentsply Maillefer, Ballaigues, Switzerland) on a 16: 1 contra-angle handpiece attached to an electric motor (X-Smart Endodontic Rotary Motor, Dentsply Sirona, United States) at $350 \mathrm{rpm}$. Preparation was carried out according to the manufacturer's recommendations. In brief, after achieving a glide path with a size $10 \mathrm{~K}$-file, the $\mathrm{X} 1$ to $\mathrm{X} 3$ files were sequentially taken to full working length. EDTA cream (17\%) (MD-ChelCream Meta Biomed, Korea) was used as a chelating agent and introduced in the canal on the tip of each successive instrument. Each set of ProTaper Next files was used to prepare 5 teeth. The canals were irrigated between files with $3 \mathrm{~mL}$ of $2.5 \% \mathrm{NaOCl}$, using $3 \mathrm{~mL}$ disposable plastic syringes with 27-gauge needle tips. This tip was placed passively into the canal, up to $3 \mathrm{~mm}$ from the apical foramen without binding. After instrumentation was completed, the root canal was irrigated with $5 \mathrm{~mL}$ of $2.5 \% \mathrm{NaOCl}$, activated with an ultrasonic file \#15 for 60 seconds, followed by irrigation with $5 \mathrm{~mL}$ of saline, then $1 \mathrm{~mL}$ of $17 \%$ EDTA solution and a final rinse with $5 \mathrm{~mL}$ saline. Paper points were used to dry the canals. The specimens were randomly divided into three sets of twelve teeth and assigned to a study group A, B, or C.

\section{Root Canal Obturation}

CWC was used to fill only the apical third of the canals. This was achieved using AH26 sealer (Dentsply Sirona, United States) and one of three different GP cones: In group A, $(n=12)$ : ProTaper Next GP cones, size X3 (Dentsply Sirona, United States); in group $\mathrm{B},(n=12)$ : ISO uniform greater taper GP cones, size 30 taper 0.04 (30/0.04 GP) (FKG Dentaire, La Chaux-de-Fonds, Switzerland), and for group C, ( $n=12$ ): nonstandardized GP cones, size medium (Meta Biomed, Korea) calibrated to size 30 using an endodontic gutta-percha calibrator and cutter (Gutta-Percha Point Gauge, Dentsply Maillefer, Ballaigues, Switzerland).

One operator fit the cones in all the canals prior to obturation according to a protocol described below. AH26 sealer mixing according to the manufacturer's instructions was carried into the canals, using size 30/0.04 GP cone dipped into the sealer and placed into the root canal with a clockwise rotational movement for 20 seconds. Then, the fitted GP cones were placed into the canals and cut at an orifice level. Another operator blinded to the groups performed CWC obturation of the apical third, using a B\&L Alpha II heat source (B\&L Biotech, United States) with a size 55/0.06 plugger that arrived $-4 \mathrm{~mm}$ of the working length. Using the plugger with a continuous heat wave $\left(180^{\circ} \mathrm{C}\right)$, the gutta-percha was softened and vertically compacted down. The teeth were stored in $37^{\circ} \mathrm{C} / 100 \%$ humidity for 72 hours before postobturation scanning.

\section{Calculating Time to Fit the Cones}

All GP cones were fitted into the canals according to the following protocol: The cone was inserted until a stop was felt, then good retention (tug-back) was assessed. If there was no tug-back, the tip of the cone was cut $0.5 \mathrm{~mm}$ at a time until a tug-back was detected. A radiograph was then taken to evaluate the cone length. If it was more than $1.5 \mathrm{~mm}$ short, a different size cone was chosen, and the radiograph was retaken to ensure that it arrived at proper working length. If the GP cone was found at or beyond the apical foramen, the radiograph was used to estimate how much to cut from the cone. After its cutting, the cone would be reinserted into the canal, tug-back reassessed, and the radiograph retaken to verify proper working length.

For each tooth, the time to fit the cone in the canal was quantified by the number of modifications necessary for a proper cone fit, with $0=$ no modifications (cone fits the length from the first trial), $1=$ one modification (cone does not fit and/or required one cut), 2 = two modifications (cone fitting required adjustment twice), etc. The number of cones used to achieve a proper cone fit as well as reasons for modification was recorded.

\section{Measurements for Sealer and Voids in Root Canal Filling Material Using Micro-CT}

A trained and calibrated micro-CT technician, blinded to the sample groups, performed micro-CT scanning and analysis. All specimens were scanned after instrumentation and postobturation. Each tooth was mounted and positioned inside the specimen chamber, 
using a customized polypropylene cylinder with a silicon material to hold the tooth. Pre- and post-obturation scans were acquired, using the Bruker SkyScan 1173 micro-CT (Bruker SkyScan, Kontich, Belgium). Scanner configuration used was $70 \mathrm{kV}$ voltage, $114 \mu \mathrm{A}$ anode current, $1000 \mathrm{~ms}$ exposure time, isotropic resolution of $14.15 \mu \mathrm{m}$ image pixel size, brass $0.25 \mathrm{~mm}, 0.4$ rotation step for $360^{\circ}$ angle, frame averaging of 4 for improved signal-to-noise ratio, and random movement of 8 , minimizing ring artifacts. A flat-field correction was performed before the scanning procedure in order to correct variations in the camera pixel sensitivity.

After scanning, reconstruction of the projected images was performed using NRecon ${ }^{\odot}$ program version 1.6.9.4. (Bruker SkyScan, Kontich, Belgium) to produce reconstructed cross-sectional images. Numerical parameters needed to establish the best image results were checked and adjusted. A ring artifact reduction of 5 for nonuniformity of the background image taken by the X-ray camera; $25 \%$ beam hardening compensation to prevent the specimen from appearing artificially denser at or near its surface and less dense at its central parts; and a smoothing of 2 using Gaussian kernel were applied. Images were saved in a 16-bit TIF file format because of the variety of specimen densities. Reconstructed images were 3D registered and loaded in the DataViewer ${ }^{\circledR}$ program version 1.5.6.2 (Bruker SkyScan, Kontich, Belgium) software to define image quality. Using $\mathrm{CTAn}^{\odot}$ program version 1.17.7.2 (Bruker SkyScan, Kontich, Belgium), the apical $4 \mathrm{~mm}$ region of the canal was identified, then $2 \mathrm{D} / 3 \mathrm{D}$ analyses were performed by selective thresholding to binarize images and give a volume value. The volume of voids was calculated using the following formula: ${ }^{24}$

Volume of voids $(\mathrm{Vv})=$ Canal (apical $4 \mathrm{~mm}$ ) volume $(\mathrm{Vc})$ - obturation (apical $4 \mathrm{~mm}$ ) volume (Vm).

Percentage of voids as well as percentage of sealer were calculated using the following formulas:

$$
\mathrm{V} v \%=\mathrm{V} v \times 100 / \mathrm{Vc}
$$

Sealer percentage $\%=\mathrm{Vm} \times 100 /$ sealer volume

Statistical analysis was conducted using JMP software version 11.1.1 (SAS Institute, Cary, NC) applying Wilcoxon/Kruskal-Wallis tests, as well as the Freeman-Halton extension of Fisher's exact test for the number of modifications to fit the cone. The level of significance was set at $p<0.05$.

\section{Results}

Voids were found in all canals regardless of GP cone type (Fig. 1). Figures $1 \mathrm{~A}$ to $\mathrm{D}$ show an obturation of canal apical third with a
ProTaper Next GP cone, while Figures $1 \mathrm{E}$ to $\mathrm{H}$ are those of an ISO uniform greater taper cone obturation and Figures $1 \mathrm{I}$ to $\mathrm{L}$ are nonstandardized GP cone obturation. The volume of voids in the apical third is represented in (Fig. 2). Using ProTaper Next GP cones resulted in a lower volume $\left(0.0570 \mathrm{~mm}^{3}\right)$ and percentage of voids (1.58\%) compared to group B $\left(0.0875 \mathrm{~mm}^{3} ; 1.93 \%\right)$ and group $C$ $\left(0.0564 \mathrm{~mm}^{3} ; 1.69 \%\right)$. This, however, was not statistically significant $(p=0.666),(p=0.379)$ (Tables 1 and 2). The mean percentage of sealer and standard deviation were $2.422( \pm 2.236)$ for group $A, 0.738$ $( \pm 0.633)$ for group $B$, and $1.219( \pm 1.646)$ for group $C$ (Fig. 3). The mean percentage of sealer in group $A$ was $2.4 \%$, compared to $0.7 \%$ in group $B$ and $1.2 \%$ in group C. Using 30/0.04 GP cones resulted in a

Table 1: Wilcoxon/Kruskal-Wallis with Chi-square approximation for void volume

\begin{tabular}{llllll}
\hline Group & $\begin{array}{l}\text { Gutta-percha } \\
\text { cone }\end{array}$ & $\begin{array}{l}\text { Score } \\
\text { sum }\end{array}$ & $\begin{array}{l}\text { Score } \\
\text { mean }\end{array}$ & $\begin{array}{l}\text { (Mean- } \\
\text { mean0)/Std0 }\end{array}$ & Prob $>\chi^{2}$ \\
\hline A & ProTaper Next & 201.000 & 16.7500 & -0117 & 0.6662 \\
B & $30 / 0.04$ & 247.000 & 20.5833 & 0.822 & \\
C & Medium & 218.000 & 18.1667 & -0.688 & \\
\hline
\end{tabular}

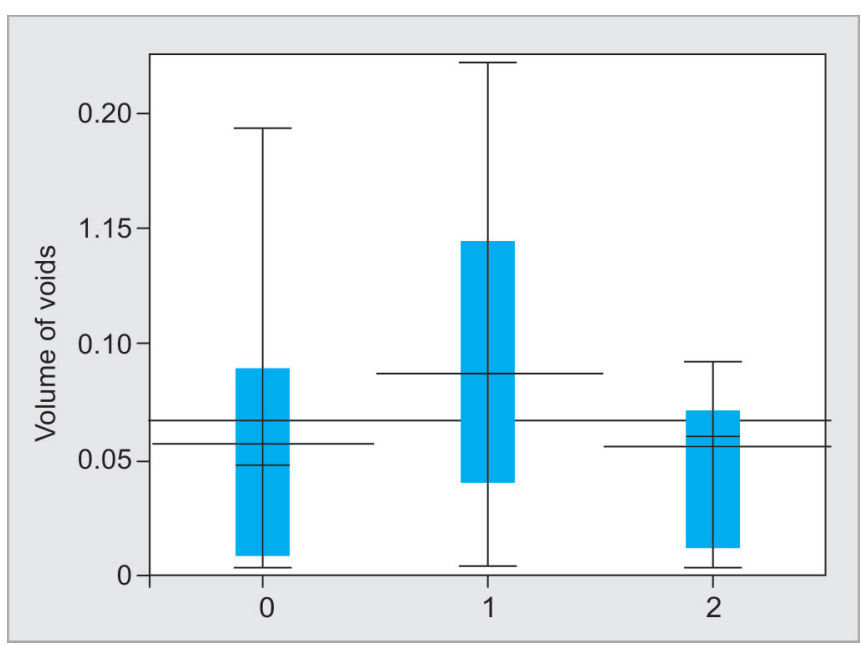

Fig. 2: Volume of voids in the apical third according to the three types of gutta-percha (GP) cones: 0 represents group A (ProTaper Next), 1 represents group $B$ (ISO uniform greater taper), and 2 represents group $C$ (nonstandardized GP). Horizontal lines represent the means for each group
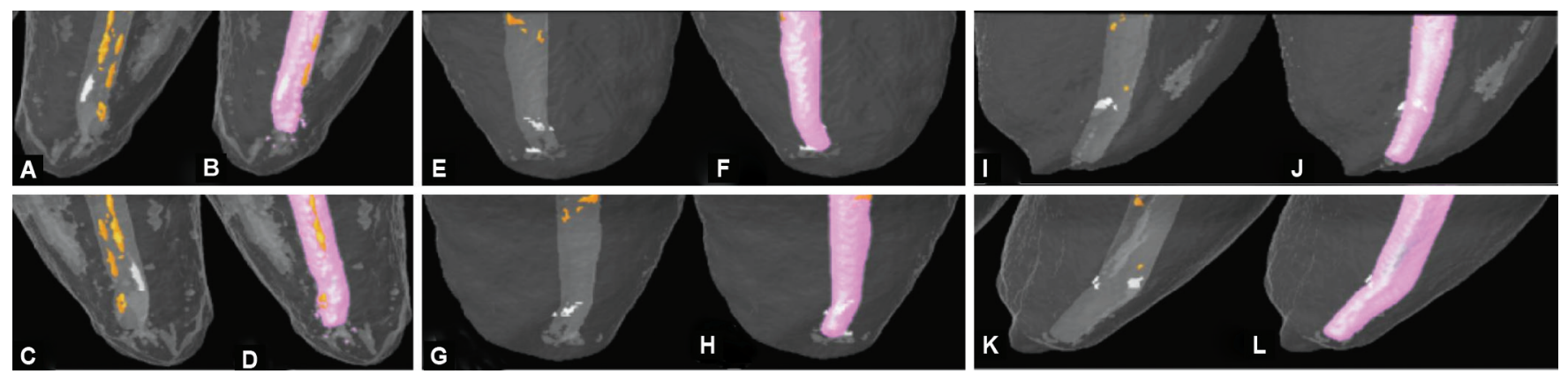

Figs. 1 A to L: The 3D visualization of canals' apical third through color-coded images. (A-D) ProTaper next GP cone obturation, (E-H) ISO uniform greater taper cone obturation, and (I-L) nonstandardized GP cone obturation. (Pink represents gutta-percha (GP), orange is sealer, and white is voids) 
Table 2: Wilcoxon/Kruskal-Wallis with Chi-square approximation for percentage of voids

\begin{tabular}{llllll}
\hline Group & $\begin{array}{l}\text { Gutta-percha } \\
\text { cone }\end{array}$ & $\begin{array}{l}\text { Score } \\
\text { sum }\end{array}$ & $\begin{array}{l}\text { Score } \\
\text { mean }\end{array}$ & $\begin{array}{l}\text { (Mean- } \\
\text { Mean0)/Std0 }\end{array}$ & Prob $>\chi^{2}$ \\
\hline A & ProTaper Next & 196.000 & 16.3333 & -0.856 & 0.3794 \\
B & $30 / 0.04$ & 263.000 & 21.9167 & 1.359 & \\
C & Medium & 207.000 & 17.2500 & -0.487 & \\
\hline
\end{tabular}

Table 3: Wilcoxon/Kruskal-Wallis with Chi-square approximation for percentage of sealer

\begin{tabular}{llllll}
\hline Group & $\begin{array}{l}\text { Gutta-percha } \\
\text { cone }\end{array}$ & $\begin{array}{l}\text { Score } \\
\text { Sum }\end{array}$ & $\begin{array}{l}\text { Score } \\
\text { mean }\end{array}$ & $\begin{array}{l}\text { (Mean- } \\
\text { Meano)/Std0 }\end{array}$ & Prob $>\chi^{2}$ \\
\hline A & ProTaper Next & 292.000 & 24.3333 & 2.332 & $0.0483^{*}$ \\
B & $30 / 0.04$ & 168.000 & 14.0000 & -1.795 & \\
C & Medium & 206.000 & 17.1667 & -0.520 & \\
\hline *Statistically significant $(p<0.05)$ & & &
\end{tabular}

Table 4: Nonparametric comparisons for each pair using Wilcoxon method

\begin{tabular}{|c|c|c|c|c|c|c|c|c|}
\hline Gutta-percha cone & Gutta-percha cone & Score mean dif & Std err dif & $Z$ & $p$-Value & Hodges-Lehmann & Lower CL & Upper CL \\
\hline Medium & $30 / 0.04$ & 2.0833 & 2.886751 & 0.72169 & 0.4705 & 0.20980 & -0.36234 & 1.14107 \\
\hline Medium & ProTaper Next & -4.7500 & 2.886751 & -1.64545 & 0.0999 & -0.78570 & -2.13701 & 0.22621 \\
\hline $30 / 0.04$ & ProTaper Next & -6.7500 & 2.886751 & -2.33827 & $0.0194^{*}$ & -1.24579 & -2.22319 & -0.15447 \\
\hline
\end{tabular}

*Statistically significant $(p<0.05)$

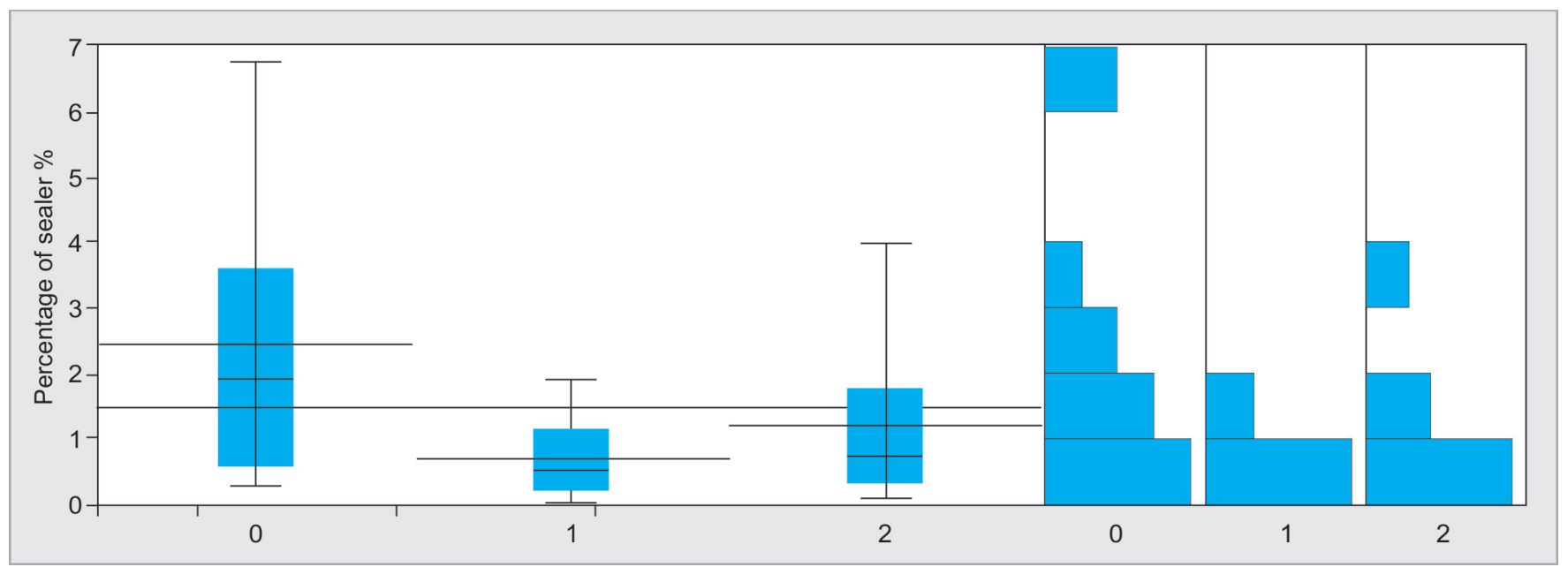

Fig. 3: Percentage of sealer in the apical third according to the three types of gutta-percha cones: 0 represents group A (ProTaper Next), 1 represents group B (ISO uniform greater taper), and 2 represents group C (nonstandardized). Horizontal lines represent the means for each group

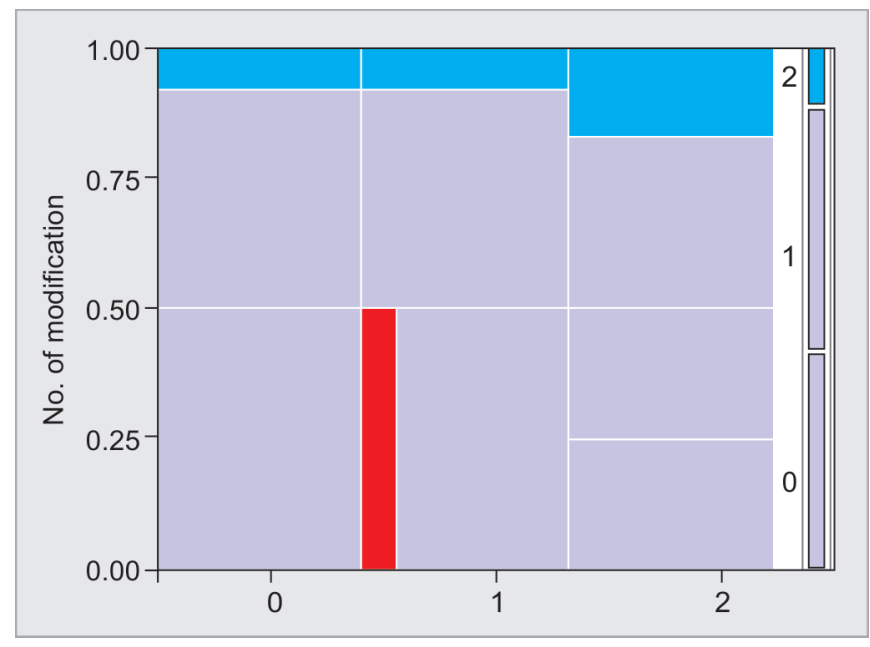

Fig. 4: Association between the number of cone modifications necessary for a proper fit in the canal and type of gutta-percha cones: 0 represents group A (ProTaper Next), 1 represents group B (ISO uniform greater taper), and 2 represents group $C$ (nonstandardized) significantly lower percentage of sealer when compared to ProTaper Next GP cones ( $p=0.019)$, but there was no statistical difference between the other groups (Tables 3 and 4).

In all groups, proper cone adaptation did not require replacement with a different size GP cone. For some canals, however, it was necessary to cut the cone to adjust the length and/ or achieve a tug-back (modification). The number of modifications necessary for proper GP cone adaptation in all groups ranged from 0 to 2, as shown in (Fig. 4). Fisher's-Freeman-Halton test revealed no significant difference between the groups $(p=0.757)$ in terms of the number of modifications.

Based on these results, we accept the null hypothesis that there are no differences between the three types of cones when used with the CWC technique, with the minor exception of sealer percentage, where the 30/0.04 nonmatching GP cones showed better results compared to the matching ProTaper Next GP cones.

\section{Discussion}

The present study revealed that system matching (ProTaper Next) GP cones, as well as nonsystem matching (ISO uniform greater taper 
and nonstandardized) GP cones were equally efficient, filling the apical third using CWC technique in terms of quality of obturation and cone adaptation, with the exception that ISO uniform greater taper cones were associated with a lower percentage of sealer. It is important for root canal obturation to effectively entomb the resistant microorganisms within the root canal, preventing them or their toxins from filtering into the periapical tissues, where they perpetuate periapical diseases. ${ }^{25}$ For this reason, the presence of voids within the apical third of the obturation may play a critical role in facilitating this exit route, leading to an endodontic treatment failure. ${ }^{22}$ This exit passage can also be promoted by the shrinkage of most sealers used to fill the root canals, and their dissolution over time with cellular fluid. ${ }^{26}$ Therefore, a large amount of sealer may lead to root canal failure, while a high percentage of gutta-percha is an indication of a better quality of obturation. Accordingly, this study evaluated the obturation quality in terms of void volume, void percentage, and sealer percentage using micro-CT, a highly accurate method that allows nondestructive three-dimensional analysis with high qualitative and quantitative correlation to histological examination, ${ }^{27,28}$ which can at the same time differentiate between gutta-percha and sealer within the root canal obturation. Only obturation of the apical third was carried out and evaluated since the rest of the canal is usually filled with injectable ( $\alpha$-phase) gutta-percha, which is generally not a GP cone type-dependent technique.

None of the obturations in this study were void free, which is in accordance with all current studies using micro-CT to compare different obturation techniques and/or root canal filling materials. ${ }^{1,4,18-24}$ Although the ProTaper Next GP group produced the lowest volume and percentage of voids, this did not prove to be statistically significant compared to other groups. No study has looked at the influence of GP cone type when using CWC. However, in a study comparing different techniques (cold lateral condensation, CWC, and single cone techniques) using greater taper and standardized 0.02 taper GP cones, Schäfer et al. also found no difference between the groups in terms of void volume and percentage. ${ }^{14}$ Studies comparing the use of matching cones with the single cone technique and CWC have also found no difference in terms of void volume and percentage. 19,21,22,24

Sealer percentage was found to be the lowest in the 30/0.04 GP group; although this was not statistically significant when compared to the nonstandardized GP group, it was significant when compared to the ProTaper Next GP group. These results seem in accordance with a study by Schäfer et al. that found that GP cones with uniform greater taper produced a lower percentage of sealer at the apical levels compared to cones with a variable greater taper (ProTaper) when used with the single cone technique. ${ }^{14}$ Another study, ${ }^{15}$ however, found that the twisted file adaptive cone, which is a uniform greater taper cone, was associated with a higher percentage of sealer compared to variable taper cones (ProTaper Universal and ProTaper Next) when also used with the single cone technique. Studies that have looked at the influence of different cone types on the percentage of GP and sealer in the apical third when using cold lateral condensation technique concluded that cone type did not influence the percentage of sealer in the canal. ${ }^{12,16}$ Although Rodrigues et al., whose study was conducted on curved mesial canals of the mandibular molars, found that the percentage of GP obtained using matching (ProTaper) GP cones was superior to that achieved using 0.02 taper GP cones, ${ }^{29}$ Lee and Kim also found that nonstandardized GP cones resulted in a significantly lower percentage of sealer compared to 0.02 taper GP cones. ${ }^{30}$

ProTaper Next files were selected in this study, due to their superior ability to shape canals and maintain the apical constriction when compared to the ProTaper Universal and the WaveOne. ${ }^{10}$ Even undergraduate students showed preference to using ProTaper Next files and were able to prepare simulated canals in significantly less time and with less procedural errors (ledges) compared to using ProTaper Universal. ${ }^{31}$

In this study, the time to fit the cone in the canal was measured in terms of the number of modifications (cutting of the cone) necessary to properly fit the cone within the canal to the required length. ProTaper Next and 30/0.04 GP cones scored the same in terms of the number of modifications. Although adapting nonstandardized GP cones required more modifications, it was not statistically significant. However, the adaptation of these nonstandardized, feathered, tipped GP cones needs manual adjustment with GP calibers and cutters, ${ }^{9}$ which incurs extra time not accounted for in this study, which may render this type of cone less efficient, compared to the ISO uniform greater taper and matching (ProTaper Next) GP cones which require no such calibration. Recent evidence has cast doubt on the degree greater taper GP cones conform to the dimensions of the NiTi instruments they correspond to, ${ }^{32-35}$ this could explain the overall lack of differences between the groups. ${ }^{32-35}$

A limitation of the present study was that it assessed the quality of obturation in an ex vivo setting, based on the percentage of voids and sealer. Further investigation is necessary to understand how this correlates clinically. Another limitation is that it was carried out on straight canals, so further research is required to extend the results to curved canals. Finally, sample size, which was calculated based on studies assessing the quality of obturation using micro-CT to measure the percentage of voids and sealer, may have been small to compare the number of modifications. Studies employing a larger sample size should be considered in that regard.

Within the limitations of this study, CWC using ProTaper Next, 30/0.04 and nonstandardized GP cones produced equivalent results in terms of obturation quality and time required for cone adaptation, with the exception of 30/0.04 GP cones, which were associated with a lower percentage of sealer.

\section{CONCLUSION}

In straight canals, using GP cones that match the NiTi instrumentation system with the CWC technique was not associated with a significantly better obturation quality in the apical third or saving of money-costing time to fit the cone within the canal compared to the ISO uniform greater taper GP cones and nonstandardized GP cones. However, further studies should be done to better understand the clinical manifestations of these ex vivo results.

\section{Clinical Significance}

When obturating straight canals using the CWC technique, GP cones that do not match the NiTi instrumentation system are as efficient as GP cones that match the NiTi instrumentation system in terms of obturation quality and ease of cone fit.

\section{Acknowledgments}

This research was funded by the Deanship of Scientific Research at PNU through the Fast-track Research Funding Program. 
Ethics: Ethical approval for the study was obtained from PNU Institutional Review Board.

Authorship Declaration: We confirm that the manuscript has been read and approved by all the named authors and that there are no other persons who satisfied the criteria for authorship but are not listed. We further confirm that the order of authors listed in the manuscript has been approved by all of us.

\section{References}

1. Hammad M, Qualtrough A, Silikas N. Evaluation of root canal obturation: a three-dimensional in vitro study. J Endod 2009;35(4):541544. DOI: 10.1016/j.joen.2008.12.021.

2. Schilder H, Goodman A, Aldrich W. The thermomechanical properties of gutta-percha. Part V. Volume changes in bulk gutta-percha as a function of temperature and its relationship to molecular phase transformation. Oral Surg Oral Med Oral Pathol 1985;59(3):285-296. DOI: 10.1016/0030-4220(85)90169-0.

3. Silver GK, Love RM, Purton DG. Comparison of two vertical condensation obturation techniques: Touch 'n heat modified and System B. Int Endod J 1999;32(4):287-295. DOI: 10.1046/j.13652591.1999.00215.x.

4. Keleş A, Alcin H, Kamalak A, et al. Micro-CT evaluation of root filling quality in oval-shaped canals. Int Endod J 2014;47(12):1177-1184. DOI: 10.1111/iej.12269.

5. Olczak K, Klimek L, Pawlicka H. Ex vivo area-metric analysis of root canal obturation using cold and warm gutta-percha. Adv Mater Sci Eng 2016;2016:6308362. DOI: 10.1155/2016/6308362.

6. van der Borden WG, Wu M-K, Wesselink PR. Percentages of guttapercha-filled canal area observed after increased apical enlargement. J Endod 2010;36(1):139-142. DOI: 10.1016/j.joen.2009.08.011.

7. Lu Q, Xing W, Peiling W. Effects of three root canal filling methods on lateral root canal filling in first mandibular premolars: an in vitro study. Chin J Conserv Dent 2012;(22):379-381. Available from: https:// en.cnki.com.cn/Article_en/CJFDTotal-YTYS201207005.htm.

8. Bal AS, Hicks ML, Barnett F. Comparison of laterally condensed .06 and .02 tapered gutta-percha and sealer in vitro. J Endod 2001;27(12):786-788. DOI: 10.1097/00004770-200112000-00019.

9. Gambarini G, Plotino G, Grande NM, et al. Matching gutta-percha cones to NiTi rotary instrument preparations. Roots 2016;2:26-28. Available from: https://www.semanticscholar.org/paper/Matchinggutta-percha-cones-to-NiTi-rotary-Gambarini-Plotino/4a1fea581ffaf 2ec58283d67bbea75f2621f8194.

10. Wu H, Peng C, Bai Y, et al. Shaping ability of ProTaper universal, waveone and protaper next in simulated L-shaped and S-shaped root canals. BMC Oral Health 2015;15:27. DOI: 10.1186/s12903-015-0012-z.

11. Khasnis SA, Kar PP, Kamal A, et al. Rotary science and its impact on instrument separation: a focused review. J Conserv Dent 2018;21(2):116-124. DOI: 10.4103/JCD.JCD_240_17.

12. Romania $C$, Beltes $P$, Boutsioukis $C$, et al. Ex-vivo area-metric analysis of root canal obturation using gutta-percha cones of different taper. Int Endod J 2009;42(6):491-498. DOI: 10.1111/j.1365-2591.2008. 01533.x.

13. Souq. Dental: online dental supplies store in Saudi Arabia. 2020. Available from: https://souq.dental/. Accessed 8 August 2020.

14. Schäfer E, Köster M, Bürklein S. Percentage of gutta-percha-filled areas in canals instrumented with nickel-titanium systems and obturated with matching single cones. J Endod 2013;39(7):924-928. DOI: 10.1016/j.joen.2013.04.001.

15. Capar ID, Ertas H, Ok E, et al. Comparison of single cone obturation performance of different novel nickel-titanium rotary systems. Acta Odontol Scand 2014;72(7):537-542. DOI: 10.3109/00016357.2013.876554.

16. Schäfer E, Nelius B, Bürklein S. A comparative evaluation of guttapercha filled areas in curved root canals obturated with different techniques. Clin Oral Investig 2012;16(1):225-230. DOI: 10.1007/ s00784-011-0509-z.
17. Banton C.Efficiency DefiNiTion. Investopedia.2020. Available from:https:// www.investopedia.com/terms/e/efficiency.asp. Accessed 8 August 2020.

18. Kabini SN, Moodley D, Parker ME, et al. An in-vitro comparative micro computed evaluation of three obturation systems. S Afr Dent J 2018;73:298-302. Available from: http://www.scielo.org.za/scielo. php?script=sci_arttext\&pid=S0011-85162018000400010\&lng=en.

19. Alshehri M, Alamri HM, Alshwaimi E, et al. Micro-computed tomographic assessment of quality of obturation in the apical third with continuous wave vertical compaction and single match taper sized cone obturation techniques. Scanning 2016;38(4):352-356. DOI: 10.1002/sca.21277.

20. Zogheib C, Hanna M, Pasqualini D, et al. Quantitative volumetric analysis of cross-linked gutta-percha obturators. Ann Stomatol (Roma) 2016;7(3):46-51. DOI: 10.11138/ads/2016.7.3.046.

21. Başer Can ED, Keleş A, Aslan B. Micro-CT evaluation of the quality of root fillings when using three root filling systems. Int Endod J 2017;50(5):499-505. Doi: 10.1111/iej.12644.

22. Angerame $D$, De Biasi $M$, Pecci $R$, et al. Analysis of single point and continuous wave of condensation root filling techniques by microcomputed tomography. Ann Ist Super Sanita 2012;48(1):35-41. DOI: 10.4415/ANN_12_01_06.

23. Somma F, Cretella G, Carotenuto M, et al. Quality of thermoplasticized and single point root fillings assessed by micro-computed tomography. Int Endod J 2011;44(4):362-369. DOI: 10.1111/j.13652591.2010.01840.x.

24. Iglecias EF, Freire LG, de Miranda Candeiro GT, et al. Presence of voids after continuous wave of condensation and single-cone obturation in mandibular molars: a micro-computed tomography analysis. $J$ Endod 2017;43(4):638-642. DOI: 10.1016/j.joen.2016.11.027.

25. Saunders WP, Saunders EM. Coronal leakage as a cause of failure in root-canal therapy: a review. Endod Dent Traumatol 1994;10(3):105108. DOI: 10.1111/j.1600-9657.1994.tb00533.x.

26. Kazemi RB, Safavi KE, Spångberg LS. Dimensional changes of endodontic sealers. Oral Surg Oral Med Oral Pathol 1993;76(6):766771. DOI: 10.1016/0030-4220(93)90050-e.

27. Jung $M$, Lommel $D$, Klimek J. The imaging of root canal obturation using micro-CT. Int Endod J 2005;38(9):617-626. DOI: 10.1111/j.13652591.2005.00990.x.

28. Kierklo A, Tabor Z, Petryniak R, et al. Application of microcomputed tomography for quantitative analysis of dental root canal obturations. Postepy Hig Med Dosw (Online) 2014;68:310-315. DOI: 10.5604/17322693.1095271.

29. Rodrigues A, Bonetti-Filho I, Faria G, et al. Percentage of guttapercha in mesial canals of mandibular molars obturated by lateral compaction or single cone techniques. Microsc Res Tech 2012;75(9):1229-1232. Available from: https://onlinelibrary.wiley.com/ doi/abs/10.1002/jemt.22053.

30. Lee E-A, Kim S-K. Obturation efficiency of non-standardized guttapercha cone in curved root canals prepared with 0.06 taper nickeltitanium instruments. J Korean Acad Conserv Dent 2005;30(2):79-84. DOI: 10.5395/JKACD.2005.30.2.079.

31. Alemam AAH, Dummer PMH, Farnell DJJ. A comparative study of protaper universal and protaper next used by undergraduate students to prepare root canals.J Endod 2017;43(8):1364-1369.DOI: 10.1016/j.joen.2017.03.038.

32. Chesler MB, Tordik PA, Imamura GM, et al. Intramanufacturer diameter and taper variability of rotary instruments and their corresponding guttapercha cones.J Jndod 2013;39(4):538-541.DOI: 10.1016/j.joen.2012.12.029.

33. Bueno J, de Melo TAF, Kunert GG. Evaluation of the tip of standardized D0 gutta-percha cones of four rotary systems, by means of an endodontic ruler. Rev Gaúcha Odontol 2017;65(4):299-302. DOI: 10.1590/1981-863720170002000023168.

34. Mirmohammadi H, Sitarz M, Shemesh H. Intra-manufacture diameter variability of rotary files and their corresponding gutta-percha cones using laser scan micrometre. Iran Endod J 2018;13(2):159-162. DOI: 10.22037/iej.v13i2.14710.

35. Haupt F, Seidel M, Rizk M, et al. Diameter and taper variability of singlefile instrumentation systems and their corresponding gutta-percha cones. J Endod 2018;44(9):1436-1441. DOI: 10.1016/j.joen.2018.06.005. 